\title{
Nonparametric subspace identification of nonlinear structures - Application to a spacecraft
}

\author{
J.P. Noël and G. Kerschen \\ Space Structures and Systems Lab, University of Liège, Belgium \\ Chemin des chevreuils 1 (B52/3), 4000, Liège, Belgium
}

\begin{abstract}
The objective of the present paper is to address the identification of a strongly nonlinear satellite structure by exploiting a new nonlinear subspace identification method formulated in the frequency domain, referred to as the FNSI method. The modal parameters of the underlying linear structure and the coefficients of the nonlinear internal forces will be estimated by this approach. Moreover, because a priori knowledge about nonlinear behaviour may be limited, the paper also proposes a nonparametric spline-based modelling strategy, encapsulated in the FNSI method. The application of interest is the SmallSat spacecraft developed by EADS-Astrium, which possesses an impact-type nonlinear device consisting of eight mechanical stops limiting the motion of an inertia wheel mounted on an elastomeric interface.
\end{abstract}

\section{Introduction}

The identification of linear and time-invariant systems is a discipline that has evolved considerably during the last forty years. The techniques available today are really quite sophisticated and advanced. In particular, subspace methods ${ }^{1}$ are powerful identification tools that are naturally applicable to multi-input multi-output (MIMO) systems. They are routinely used for experimental modal analysis, ${ }^{2}$ but also for advanced processing such as damage detection and structural health monitoring. ${ }^{3}$ However, because engineering structures are known to be prone to nonlinearity, there is a crucial need for extending linear subspace methods to a practical nonlinear analogue. In this context, the first contribution is due to Lacy and Bernstein, ${ }^{4}$ who derived a time-domain algorithm applicable to nonlinear mechanical systems, later improved by Marchesiello and Garibaldi. ${ }^{5}$ More recently, a dual approach has been developed in the frequency domain, referred to as the frequency-domain nonlinear subspace identification (FNSI) method, ${ }^{6}$ whose main advantage is the possibility to discriminate frequency samples according to information content and signal-to-noise ratio, thereby reducing the computational burden.

The objective of the present paper is to address the identification of a strongly nonlinear satellite structure by exploiting the FNSI method. The modal parameters of the underlying linear structure and the coefficients of the nonlinear internal forces will be estimated by this approach. Moreover, because a priori knowledge about nonlinear behaviour may be limited, the paper also proposes a nonparametric spline-based modelling strategy, encapsulated in the FNSI method. The application of interest is the SmallSat spacecraft developed by EADS-Astrium, which possesses an impact-type nonlinear device consisting of eight mechanical stops limiting the motion of an inertia wheel mounted on an elastomeric interface. This application is challenging for several reasons including the non-smooth nature of the nonlinear component, high modal density and high non-proportional damping.

The paper is organised as follows. Section II introduces the theoretical background of the FNSI method. The SmallSat spacecraft is then described in Section III and details about its finite element modelling are given. Section IV next addresses the identification of the SmallSat structure based on two synthetic data sets corrupted by noise. In the first numerical experiment, the excitation is chosen such that a single nonlinearity is activated and its identification is discussed via two sets of processed channels and is supported by the use of stabilisation diagrams. A nonparametric approach to nonlinearity characterisation based on cubic splines 
is then proposed and is proved to yield a reliable modelling of the nonlinear mechanism. A second numerical experiment is then analysed involving the strong activation of all WEMS nonlinearities that are shown to be accurately identified by the FNSI method coupled with the use of cubic splines. Finally, the conclusions of the study are summarised in Section V.

\section{Subspace identification of nonlinear structures in the frequency domain}

As described in the review paper written by Kerschen et al. ${ }^{7}$ the identification of nonlinear mechanical systems can be seen as a progression through nonlinearity detection, characterisation and parameter estimation. Following the detection step whose goal is obvious, characterisation is concerned with nonlinearity localisation and model selection. The model parameters are then estimated, for example, by least-squares fitting or nonlinear optimisation depending upon the method considered. The aim of the FNSI method is to address this latter step and so to estimate nonlinear stiffness and damping coefficients together with the frequency response function (FRF) matrix of the underlying linear system.

\section{II.A. State-space model and problem statement}

The vibrations of nonlinear systems are governed by the time-continuous model

$$
M \ddot{q}(t)+C_{v} \dot{q}(t)+K q(t)+f(q(t), \dot{q}(t))=p(t)
$$

where $M, C_{v}, K \in \mathbb{R}^{r \times r}$ are the linear mass, viscous damping and stiffness matrices, respectively; $q(t)$ and $p(t) \in \mathbb{R}^{r}$ are the generalised displacement and external force vectors, respectively; $f(t) \in \mathbb{R}^{r}$ is the nonlinear restoring force vector, and $r$ the number of degrees of freedom (DOFs) of the structure obtained after spatial discretisation. The amplitude, direction, location and frequency content of the excitation $p(t)$ determine in which regime the structure behaves. The joint effect of the $s$ lumped nonlinearities in the system is modelled using a summation of the form

$$
f(q(t), \dot{q}(t))=\sum_{j=1}^{s} \mu_{j} g_{j}(q(t), \dot{q}(t)) b_{j} .
$$

Each term contains an unknown nonlinear coefficient $\mu_{j}$ and the corresponding functional form $g_{j}(t)$, which is assumed to be known. Nonlinearity localisation is specified using a vector of Boolean values, $b_{j} \in \mathbb{R}^{r}$. In the technical literature about subspace methods, first-order state-space models are preferred to the secondorder description of the dynamics in Equation (1), because of the intrinsic capability of a state-space model to encompass MIMO systems. Considering that displacements are measured and defining the state vector $x=\left(\begin{array}{ll}q^{T} & \dot{q}^{T}\end{array}\right)^{T} \in \mathbb{R}^{n}$, the equations of motion are recast into

$$
\left\{\begin{aligned}
\dot{x}(t) & =A_{c} x(t)+B_{c}^{n l} g(q(t), \dot{q}(t))+B_{c} p(t) \\
q(t) & =C_{c} x(t)+D_{c} p(t)
\end{aligned}\right.
$$

where subscript $c$ stands for continuous-time; $A_{c} \in \mathbb{R}^{n \times n}, B_{c}^{n l} \in \mathbb{R}^{n \times s}, B_{c} \in \mathbb{R}^{n \times r}, C_{c} \in \mathbb{R}^{r \times n}$ and $D_{c} \in \mathbb{R}^{r \times r}$ are the state, nonlinear coefficient, input, output and direct feed-through matrices, respectively; $g(t) \in \mathbb{R}^{s}$ gathers the basis functions $g_{j}(t)$, and $n=2 r$. State-space and physical-space matrices correspond through the relations

$$
\begin{gathered}
A_{c}=\left(\begin{array}{cc}
0^{r \times r} & \mathrm{I}^{r \times r} \\
-M^{-1} K & -M^{-1} C_{v}
\end{array}\right) \quad B_{c}^{n l}=\left(\begin{array}{cccc}
0^{r \times 1} & 0^{r \times 1} & \ldots & 0^{r \times 1} \\
-\mu_{1} M^{-1} b_{1} & -\mu_{2} M^{-1} b_{2} & \ldots & -\mu_{s} M^{-1} b_{s}
\end{array}\right) \\
B_{c}=\left(\begin{array}{c}
0^{r \times r} \\
M^{-1}
\end{array}\right) \quad C_{c}=\left(\begin{array}{ll}
\mathrm{I}^{r \times r} & 0^{r \times r}
\end{array}\right) D_{c}=0^{r \times r}
\end{gathered}
$$

where 0 and I are the zero and identity matrices, respectively.

Given measurements of $p(t)$ and $q(t)$, the FNSI method first delivers an appropriate system order $n$ and the five state-space matrices $A_{c}, B_{c}^{n l}, B_{c}, C_{c}$ and $D_{c}$. The estimation of the nonlinear coefficient $\mu_{j}$ and of the FRF matrix of the underlying linear system $H(\omega)$ is subsequently achieved by means of the conversion scheme from state space to physical space outlined in Section II.E. 


\section{II.B. Equivalent linear identification through feedback}

The FNSI method relies on the feedback interpretation ${ }^{8}$ of the dynamics governed by Equation (1), illustrated in Figure 1. It consists in moving the nonlinear forces to the right-hand side of this equation and viewing them as external forces applied to the underlying linear structure. Thus, the internal forces that are nonlinear functions of the outputs act as a feedback to the linear open-loop system. Considering Equations (3), this interpretation boils down into the concatenation of $g(t)$ and $p(t)$ into a single extended input vector $e(t) \in \mathbb{R}^{s+r}:$

$$
\left\{\begin{aligned}
\dot{x}(t) & =A_{c} x(t)+B_{c}^{e} e(q(t), \dot{q}(t), p(t)) \\
q(t) & =C_{c} x(t)+D_{c}^{e} e(t)
\end{aligned}\right.
$$

where $B_{c}^{e}=\left(\begin{array}{ll}B_{c}^{n l} & B_{c}\end{array}\right) \in \mathbb{R}^{n \times(s+r)}$ and $D_{c}^{e}=\left(\begin{array}{ll}0^{r \times s} & D_{c}\end{array}\right) \in \mathbb{R}^{r \times(s+r)}$. The feedback interpretation is particularly appealing because the inverse problem to be solved is now equivalent to the widely-studied linear state-space identification problem. However, the presence of nonlinearities in input-output data requires the interpretation and use of the FNSI algorithm to be tailored, as discussed throughout this paper.

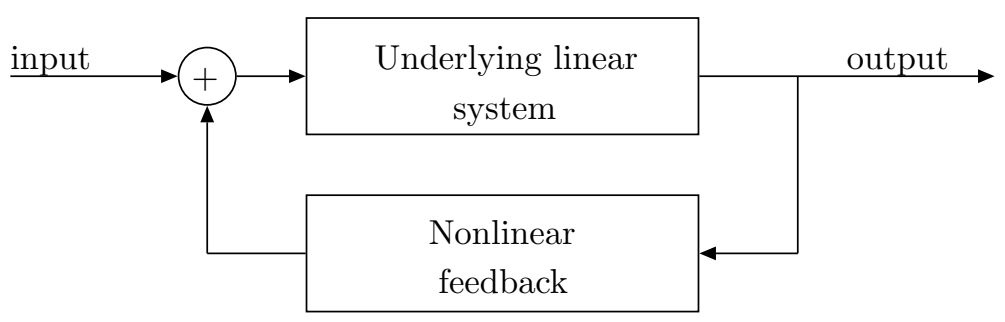

Figure 1: Feedback interpretation of nonlinear mechanical systems. ${ }^{8}$

As extensively substantiated by Pintelon and Schoukens, ${ }^{9}$ tackling system identification problems in the frequency domain is an attractive and versatile alternative. For improved numerical conditioning, ${ }^{10} \mathrm{a}$ discrete-time translation of Equations (5) is first considered, before applying the discrete Fourier transform (DFT). Provided that the time signal $v(t)$ is periodic and observed over an integer number of periods in steady-state conditions, its DFT $V(k)$ is given by

$$
V(k)=\frac{1}{\sqrt{M}} \sum_{\tau=0}^{M-1} v(\tau) e^{-j 2 \pi k \tau / M}
$$

where $M$ is the number of recorded time samples, $k$ the frequency line and $j$ the imaginary unit. Equations (5) eventually write

$$
\left\{\begin{aligned}
z_{k} X(k) & =A_{d} X(k)+B_{d}^{e} E(k) \\
Q(k) & =C_{d} X(k)+D_{d}^{e} E(k)
\end{aligned}\right.
$$

where subscript $d$ stands for discrete-time; $z_{k}=e^{j 2 \pi k / M}$ is the Z-transform variable, and $X(k), E(k)$ and $Q(k)$ the DFTs of $x(t), e(t)$ and $q(t)$, respectively.

One also points out that, in practice, only a limited set of DOFs in $p(t)$ and $q(t)$ are excited and observed, respectively. The identification problem is therefore preferably stated in terms of the measured applied forces $u(t) \in \mathbb{R}^{m \leq r}$ and displacements $y(t) \in \mathbb{R}^{l \leq r}$. Accordingly, the extended input vector is $e(y(t), \dot{y}(t), u(t)) \in \mathbb{R}^{s+m}$. Equations (7) become

$$
\left\{\begin{aligned}
z_{k} X(k) & =A_{d} X(k)+B_{d}^{e} E(k) \\
Y(k) & =C_{d} X(k)+D_{d}^{e} E(k)
\end{aligned}\right.
$$

where $Y(k)$ is the DFT of $y(t)$ and where matrices $A_{d}, B_{d}^{e}, C_{d}$ and $D_{d}^{e}$ are now a projection of the original matrices onto the observed and controlled DOFs. The subscript $d$ will be skipped afterwards, because no ambiguity is possible. 


\section{II.C. Output-state-input matrix equation formulation}

Subspace identification methods are built upon the reformulation of the state-space relations (8) in a matrix form. For this purpose, the measured output frequency spectra matrix is defined as

$$
Y_{i}=\left(\begin{array}{cccc}
Y(1) & Y(2) & \ldots & Y(N) \\
z_{1} Y(1) & z_{2} Y(2) & \ldots & z_{N} Y(N) \\
z_{1}^{2} Y(1) & z_{2}^{2} Y(2) & \ldots & z_{N}^{2} Y(N) \\
\vdots & & & \\
z_{1}^{i-1} Y(1) & z_{2}^{i-1} Y(2) & \ldots & z_{N}^{i-1} Y(N)
\end{array}\right) \in \mathbb{R}^{l i \times N}
$$

where $i$ is an user-defined index and $N$ the number of non-necessarily equidistant frequency lines exploited in the identification. Defining $\zeta=\operatorname{diag}\left(z_{1} z_{2} \ldots z_{N}\right) \in \mathbb{R}^{N \times N}, Y_{i}$ is recast into

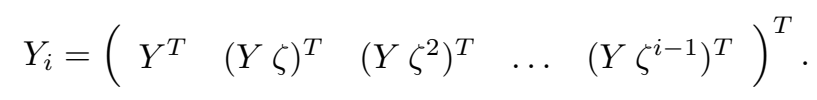

The extended input frequency spectra matrix is similarly formed as

$$
E_{i}=\left(\begin{array}{lllll}
E^{T} & (E \zeta)^{T} & \left(E \zeta^{2}\right)^{T} & \ldots & \left(E \zeta^{i-1}\right)^{T}
\end{array}\right)^{T} \in \mathbb{R}^{(s+m) i \times N} .
$$

Introducing the extended observability matrix

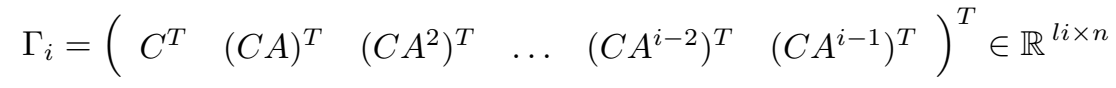

and the lower-block triangular Toeplitz matrix $\Lambda_{i}$

$$
\Lambda_{i}=\left(\begin{array}{ccccc}
D^{e} & 0 & 0 & \ldots & 0 \\
C B^{e} & D^{e} & 0 & \ldots & 0 \\
C A B^{e} & C B^{e} & D^{e} & \ldots & 0 \\
\vdots & \vdots & \vdots & & \vdots \\
C A^{i-2} B^{e} & C A^{i-3} B^{e} & C A^{i-4} B^{e} & \ldots & D^{e}
\end{array}\right) \in \mathbb{R}^{l i \times(s+m) i}
$$

recursive substitution of Equations (8) results in the output-state-input matrix equation

$$
Y_{i}=\Gamma_{i} X+\Lambda_{i} E_{i}
$$

where $X \in \mathbb{R}^{n \times N}$ is the state spectrum and where the index $i$ is explicitly written to signal the number of block rows of a matrix.

Remark that $i$ and $N$ must be chosen to encompass sufficient valuable information to identify the system. The choice of $N$ is briefly discussed in Section IV where the possibility to focus on frequency regions of interest will prove to be a major advantage of the FNSI method. A physics-based or information-based decision about $i$ is more delicate. Basically, the larger $i$, the more accurate the identification since $i$ conveys how system dynamics is included in the data matrices. However, redundant information can effect the conditioning of those matrices, hence imposing bounds to $i$. There also exists an obvious trade-off between the values of $i$ and $N$ and the time needed to inverse the model.

\section{II.D. Estimation of the system matrices}

The FNSI-based computation of the system matrices is a three-step procedure starting from Equation (14). First, an estimate of the extended observability matrix $\Gamma_{i}$ is computed. To this end, the term depending on the input and the nonlinearities in Equation (14), namely $\Lambda_{i} E_{i}$, is eliminated using a geometrical projection. Specifically, the two-dimensional interpretation of Equation (14) depicted in Figure 2 shows that an 


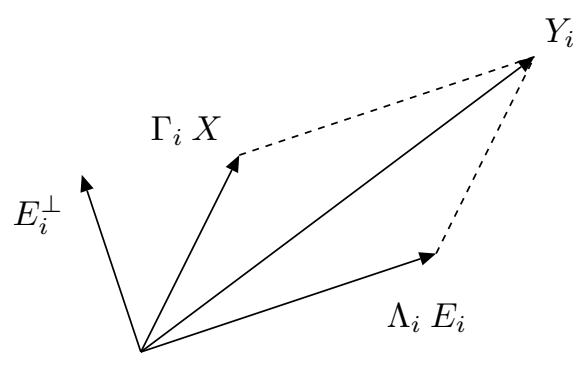

Figure 2: Geometrical interpretation of Equation (14) in a two-dimensional space.

orthogonal projection onto the orthogonal complement of $E_{i}$, denoted $E_{i}^{\perp}$, cancels the extended input term.

Matrix $\Gamma_{i}$ can then be obtained through a truncated singular value decomposition of the result of the projection. The truncation limits the singular value spectrum to genuine elements, thus removing spurious values and, in turn, reducing the influence of noise and rounding errors on the identification. In addition, the number of retained singular values yields the system order $n$. From the knowledge of $\Gamma_{i}$ and $n$, the second step consists in computing the matrices $A$ and $C$ by means of the shift property $\underline{\Gamma_{i}} A=\overline{\Gamma_{i}}$, where $\underline{\Gamma_{i}}$ and $\overline{\Gamma_{i}}$ are the matrix $\Gamma_{i}$ without the last and first $l$ rows, respectively. State matrix $A$ is consequently found as the least-squares solution of the over-determined system of equations $A=\Gamma_{i}{ }^{\dagger} \overline{\Gamma_{i}}$, where $\dagger$ is the pseudo-inverse, while output matrix $C$ is extracted from $\Gamma_{i}$ as its first block row. The final step is the estimation of the two system matrices $B^{e}$ and $D^{e}$. A robust resolution scheme that exploits the formulation of a set of linear equations in $B^{e}$ and $D^{e}$, explicitly solved in least-squares sense, has been proposed in a previous work ${ }^{6}$ and is used herein. It is however not detailed for the sake of conciseness.

\section{II.E. Conversion from state space to physical space}

Once a spate-space model $\left(A, B^{e}, C, D^{e}\right)$ is identified, the final step is the estimation of the nonlinear coefficients $\mu_{j}$ and of the FRF matrix of the underlying linear system $H(\omega)$

$$
H(\omega)=\left(-\omega^{2} M+j \omega C_{v}+K\right)^{-1} .
$$

To achieve this transformation back to physical space, one notes that, in the frequency domain, the substitution of Equation (2) into Equation (1) gives

$$
H^{-1}(\omega) Q(\omega)+\sum_{j=1}^{s} \mu_{j} G_{j}(\omega) b_{j}=P(\omega)
$$

where $Q(\omega), G_{j}(\omega)$ and $P(\omega)$ are the continuous-time Fourier transforms of $q(t), g_{j}(t)$ and $p(t)$, respectively. The concatenation of $G_{j}(\omega)$ and $P(\omega)$ further introduces the extended input spectra $E(\omega)$ so as to obtain the linear frequency-domain relationship

$$
Q(\omega)=H(\omega)\left[\begin{array}{llll}
I^{r \times r} & -\mu_{1} b_{1} & \ldots & -\mu_{s} b_{s}
\end{array}\right] E(\omega)=H^{e}(\omega) E(\omega)
$$

where the matrix $H^{e}(\omega)$ extends the concept of FRF to nonlinear mechanical systems, and encompasses both the underlying linear FRF matrix and the nonlinear coefficients. Moreover, it was proved ${ }^{5}$ that the extended FRF matrix $H^{e}(\omega)$ is an invariant system property and that it can be retrieved, similarly to linear theory, from the combination of the continuous-time state-space matrices

$$
H^{e}(\omega)=C_{c}\left(j \omega \mathrm{I}^{n \times n}-A_{c}\right)^{-1} B_{c}^{e}+D_{c}^{e} .
$$

As a result, the nonlinear coefficients identified from $H^{e}(\omega)$ using Equations (17) and (18) are spectral quantities, i.e. they are complex-valued and frequency-dependent. A reliable identification scheme together 
with an appropriate selection of the nonlinear functional forms $g_{j}(t)$ should make the imaginary parts much smaller than the corresponding real parts. The frequency dependence of the coefficients should also remain small. These indications will serve as quality criteria in Section IV.

\section{Description and modelling of the SmallSat spacecraft}

The SmallSat structure was conceived by EADS-Astrium as a low-cost platform for small satellites in low earth orbits. ${ }^{11}$ It is a monocoque tube structure which is $1.2 \mathrm{~m}$ in height and $1 \mathrm{~m}$ in width. It incorporates eight flat faces for equipment mounting purposes, creating an octagon shape, as shown in Figure 3. The octagon is manufactured using carbon-fibre-reinforced plastic by means of a filament winding process. The structure thickness is $4 \mathrm{~mm}$ with an additional $0.25-\mathrm{mm}$-thick skin of Kevlar applied to both the inside and outside surfaces to provide protection against debris. The top floor is an $1-m^{2}$ sandwich aluminium panel, with 25- $\mathrm{mm}$ core and 1- $\mathrm{mm}$ skins. The interface between the spacecraft and launch vehicle is achieved via four aluminium brackets located around cut-outs at the base of the structure. The total mass including the interface brackets is around $64 \mathrm{~kg}$.

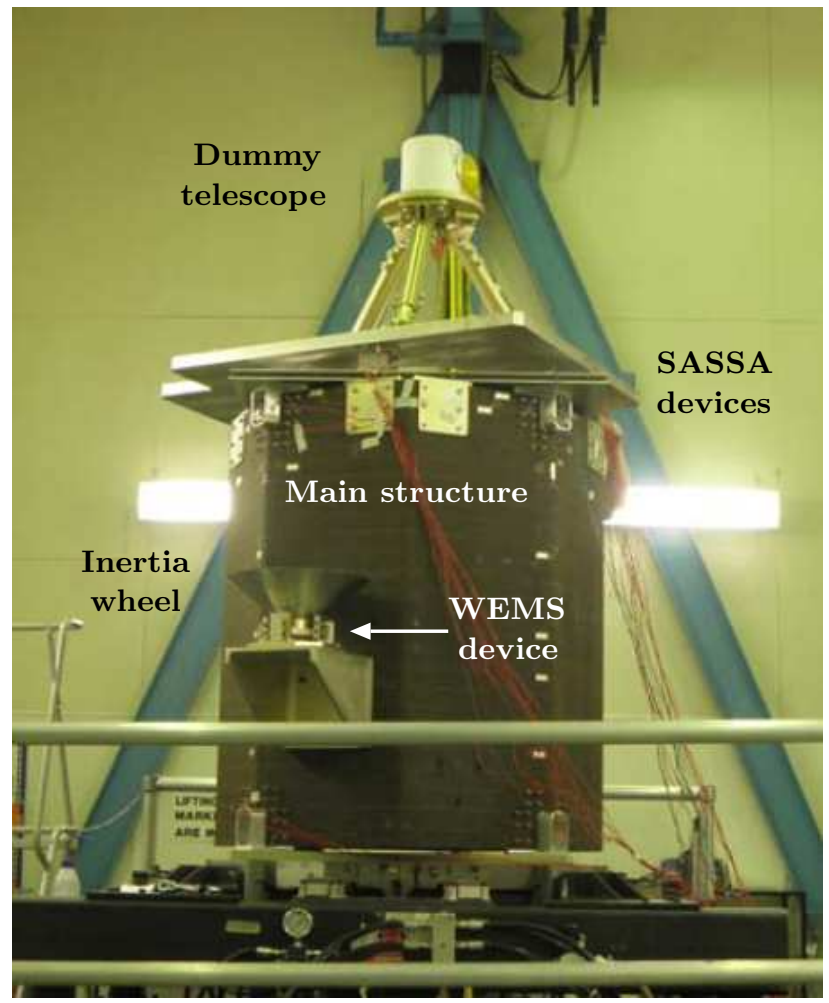

Figure 3: SmallSat spacecraft equipped with an inertia wheel supported by the WEMS device and a dummy telescope connected to the main structure by the isolating SASSA devices.

The spacecraft structure supports a dummy telescope mounted on a baseplate through a tripod; its mass is around $140 \mathrm{~kg}$. The dummy telescope plate is connected to the SmallSat top floor by three shock attenuators, termed Shock Attenuation System for Spacecraft and Adaptor (SASSA), whose behaviour is considered as linear in this study. Besides, as depicted in Figure 4 (a), a support bracket connects to one of the eight walls the so-called Wheel Elastomer Mounting System (WEMS) device which is loaded with an 8- $k g$ dummy inertia wheel. The WEMS device is a mechanical filter which mitigates disturbances coming from the inertia wheel through the presence of a soft elastomeric interface between its mobile part, i.e. the inertia wheel and a supporting metallic cross, and its fixed part, i.e. the bracket and by extension the spacecraft. Moreover, eight mechanical stops limit the axial and lateral motions of the WEMS mobile part during launch, which gives rise to strongly nonlinear dynamical phenomena. A thin layer of elastomer placed onto the stops is however used to prevent metal-metal impacts. Figure 4 (b) presents a simplified though 
relevant modelling of the WEMS device where the inertia wheel is seen as a point mass. The four nonlinear connections (NCs) between the WEMS mobile and fixed parts are labelled NC $1-4$. Each NC possesses a trilinear spring in the axial direction (elastomer in traction/compression plus two stops), a bilinear spring in the radial direction (elastomer in shear plus one stop) and a linear spring in the third direction (elastomer in shear). In Figure 4 (b), linear and nonlinear springs are denoted by squares and circles, respectively.
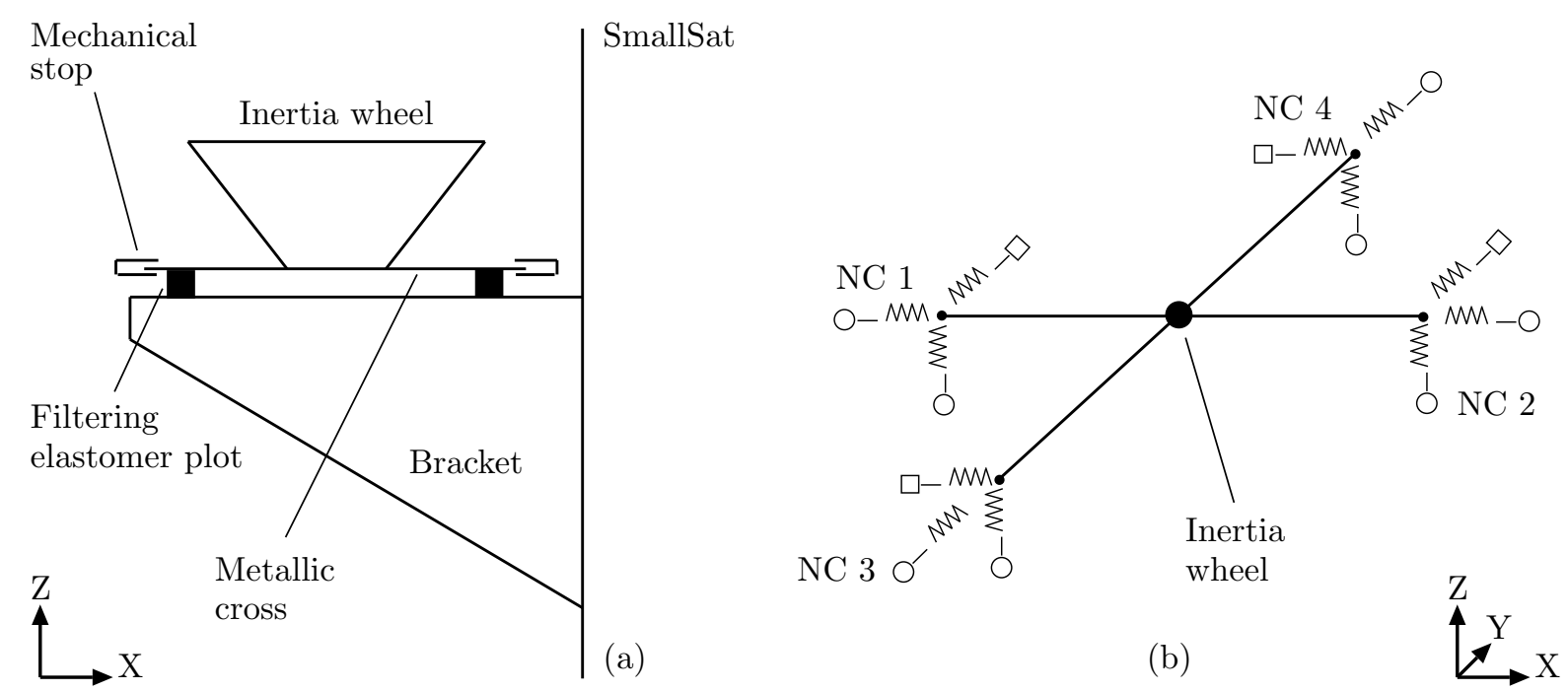

Figure 4: WEMS device. (a) Detailed description of the WEMS components; (b) simplified modelling of the WEMS mobile part considering the inertia wheel as a point mass. The linear and nonlinear connections between the WEMS mobile and fixed parts are signalled by squares and circles, respectively.

A finite element model (FEM) of the SmallSat was developed and used in the present work to conduct numerical experiments. It comprises about 65,000 DOFs and the comparison with experimental data revealed its good predictive capabilities. The model consists of shell elements (octagon structure and top floor, instrument baseplate, bracket and WEMS metallic cross) and point masses (dummy inertia wheel and telescope) and meets boundary conditions with four clamped nodes. Structural damping is considered by means of the proportional damping rule and the high dissipation in the elastomer components of the WEMS is described using lumped dashpots, hence resulting in a highly non-proportional damping matrix. Table 1 gives the natural frequencies $\omega$ and damping ratios $\epsilon$ of the first 11 modes of the SmallSat up to $50 \mathrm{~Hz}$. They are listed together with a brief description of the corresponding deformations of the structure. One remarks that the WEMS local modes likely to involve nonlinear dynamics are clustered in two groups around 10 and $30 \mathrm{~Hz}$. Subsequent resonances involve bracket bending and axial and lateral motions of the telescope supporting panel.

To achieve tractable nonlinear calculations, the linear elements of the FEM were condensed using the Craig-Bampton reduction technique. This approach consists in expressing the system dynamics in terms of some retained DOFs and internal modes of vibration. Specifically, the full-scale model of the spacecraft was reduced to 13 nodes (excluding DOFs in rotation), namely both sides of each NC (8), the inertia wheel point mass (1) and the four corners of the instrument baseplate (4), and 100 modes. In total, the reduced-order model thus contains 139 DOFs. Nonlinearity was finally introduced within the WEMS module between the NC nodes given eight piecewise-linear springs whose parameters are listed in Table 2. Time integration was carried out using a nonlinear Newmark routine with a sampling frequency of 20,000 $\mathrm{Hz}$ to capture high-frequency oscillations during impacts. Simulated time series were then decimated down to 2,500 $\mathrm{Hz}$ for practical use, considering low-pass filtering to avoid aliasing. Filtered white noise in $0-100 \mathrm{~Hz}$ was finally added to the synthetic signals to recreate the mechanical and electrical disturbances observed in a typical measurement setup. The noise level was set at $0.5 \%$ of the root-mean-square (RMS) response amplitude at the bracket tip. 


\begin{tabular}{|c|c|c|c|}
\hline Mode & $\begin{array}{c}\text { Natural } \\
\text { frequency } \omega(H z)\end{array}$ & $\begin{array}{l}\text { Damping } \\
\text { ratio } \epsilon(\%)\end{array}$ & Description \\
\hline 1 & 10.67 & 5.34 & $\begin{array}{l}\text { Concave motion of the } \\
\text { WEMS mobile part (WMP) along X }\end{array}$ \\
\hline 2 & 11.01 & 5.58 & Symmetric to mode 1 along $\mathrm{Y}$ \\
\hline 3 & 28.26 & 9.79 & Convex motion of the WMP along $\mathrm{X}$ \\
\hline 4 & 28.41 & 10.62 & Symmetric to mode 3 along Y \\
\hline 5 & 30.11 & 7.03 & $\begin{array}{l}\text { Bracket bending and } \\
\text { in-phase motion of the WMP along } \mathrm{Z}\end{array}$ \\
\hline 6 & 30.52 & 15.54 & Rotation of the WMP around Z \\
\hline 7 & 31.59 & 2.07 & Rotation of the instrument panel (IP) \\
\hline 8 & 32.62 & 2.25 & $\begin{array}{l}\text { Rotation of the IP perpendicularly to } \\
\text { mode } 7 \text { and motion of the WMP along } \mathrm{Z}\end{array}$ \\
\hline 9 & 37.47 & 2.27 & $\begin{array}{c}\text { Rotation of the IP similarly to mode } 8 \\
\text { and rotation around } \mathrm{Z}\end{array}$ \\
\hline 10 & 38.26 & 2.33 & $\begin{array}{l}\text { Main structure bending and in-plane motion } \\
\text { of the IP in phase }\end{array}$ \\
\hline 11 & 43.31 & 2.51 & Rotation of the IP similarly to mode 8 \\
\hline
\end{tabular}

Table 1: Natural frequencies $\omega$ and damping ratios $\epsilon$ of the first 11 modes of the SmallSat up to $50 \mathrm{~Hz}$ and description of the corresponding deformations of the structure.

\begin{tabular}{cccc}
\hline & Clearance & Linear slope & Nonlinear slope \\
Axial Z & 1.5 & 8 & 100 \\
Lateral X and Y & 2 & 2 & 40 \\
\hline
\end{tabular}

Table 2: Parameters of the WEMS stiffness curves given through adimensional values for confidentiality reasons.

\section{SmallSat identification based on noisy data}

This section addresses the identification of the SmallSat structure based on two synthetic data sets corrupted by additive noise. In the first numerical experiment, the excitation is chosen such that a single nonlinearity is activated and its identification is discussed via two sets of processed channels and is supported by the use of stabilisation diagrams. A nonparametric approach to nonlinearity characterisation based on cubic splines is then proposed and is proved to yield a reliable modelling of the nonlinear mechanism. A second numerical experiment is then analysed involving the strong activation of all WEMS nonlinearities that are shown to be accurately identified by the FNSI method coupled with the use of cubic splines.

\section{IV.A. Single nonlinearity case}

A periodic noise forcing with a RMS value of $100 \mathrm{~N}$ was applied in the axial direction to the NC 2 on the inertia wheel side. It consisted of a normally-distributed random signal (30,000 points over 12 seconds) repeated 8 times and band-limited in $5-50 \mathrm{~Hz}$ for a total of 240,000 points. The amplitude and the location of this excitation caused impacts exclusively in the NC 2, as confirmed in Table 3 which reports the number of clearance exceedances in the eight nonlinear springs of the WEMS device. In the application of the FNSI method, the index $i$ is set to 50 and 180,000 time samples are retained after the rejection of the 2 first periods of measurement to avoid leakage. Transforming the inverse problem into the frequency domain, only 3,240 frequency lines spanning the input band from 5 to $50 \mathrm{~Hz}$ are finally exploited. 


\begin{tabular}{ccccc}
\hline & NC 1 & NC 2 & NC 3 & NC 4 \\
$\mathrm{X}$ & 0 & 0 & - & - \\
$\mathrm{Y}$ & - & - & 0 & 0 \\
$\mathrm{Z}$ & 0 & 11,135 & 0 & 0 \\
\hline
\end{tabular}

Table 3: Number of clearance exceedances. No entry means the absence of mechanical stop.

The first step in formulating a nonlinear subspace model is the selection of an adequate model order, which translates the number of linear modes excited in the output data. This step is crucial since too low orders involve unmodeled dynamics, whereas too large orders lead to overmodelling issues such as an increase of the noise sensitivity of the model. In linear system identification, stabilisation diagrams are most frequently exploited as decision-making tools and have proved successful in numerous industrial applications. ${ }^{12}$ The capability of the FNSI method to retrieve linear system parameters from nonlinear data does still make the use of the stabilisation diagram effective in nonlinear subspace-based identification. Figure 5 charts the stabilisation of the natural frequencies, damping ratios and mode shapes of the structure for model orders up to 60. This analysis was conducted using all measured channels, i.e. $39 \mathrm{DOFs}$, as output data. Note also that the modal assurance criterion for complex-valued mode shapes (MACX), as defined by Vacher et $a l .,{ }^{13}$ is utilised in the diagram as the damping mechanisms in the SmallSat were shown to be highly nonproportional. Figure 5 shows full stabilisation of 8 modes at order 20. However, since this is tested between two successive model orders taking as reference the lowest one, equal stabilisation is also achieved at order 18 which is therefore selected to avoid spurious poles. Table 4 lists the corresponding relative errors on the estimated natural frequencies and damping ratios and the associated MACX matrix is plotted in Figure 6. They all prove the excellent quality of the linear modal properties of the spacecraft recovered by the FNSI method, except for mode 9 which however hardly participates in the system response under axial loading.

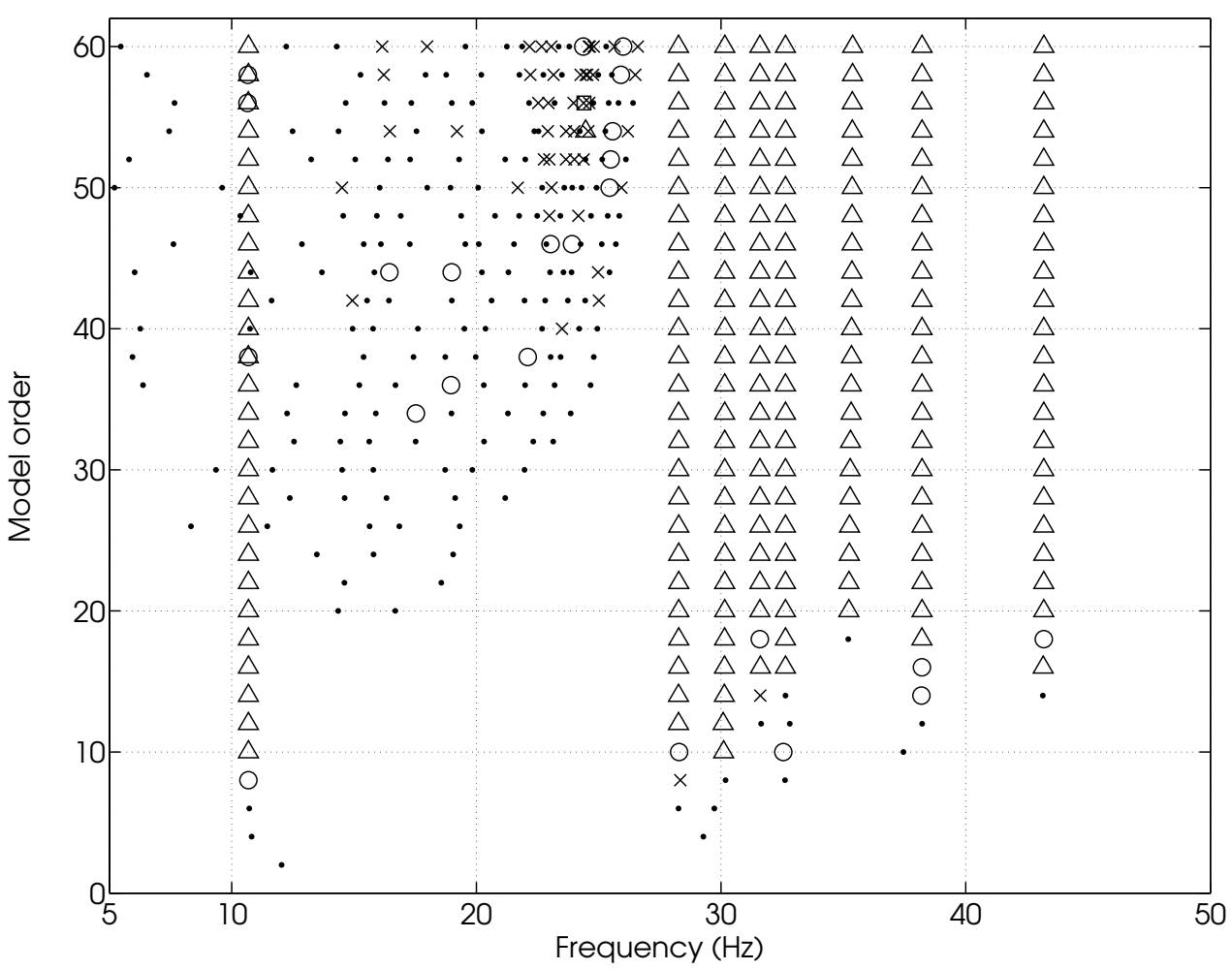

Figure 5: Stabilisation diagram computed using all measured channels. Dot: new pole; cross: stab. in natural frequency; square: extra stab. in damping ratio; circle: extra stab. in MACX; triangle: full stab.

The complex-valued nonlinear coefficient identified by FNSI is depicted in Figure 7 (a). It was estimated 


\begin{tabular}{ccc}
\hline Mode & Error on $\omega(\%)$ & Error on $\epsilon(\%)$ \\
1 & 0.16 & -0.01 \\
3 & 0.03 & -0.59 \\
5 & 0.11 & -0.37 \\
7 & -0.03 & -2.68 \\
8 & 0.03 & 0.25 \\
9 & -6.05 & -21.48 \\
10 & -0.13 & 0.68 \\
11 & -0.28 & -0.28 \\
\hline
\end{tabular}

Table 4: Relative errors on the estimated linear natural frequencies and damping ratios (in \%) computed at order 18 using all measured channels.

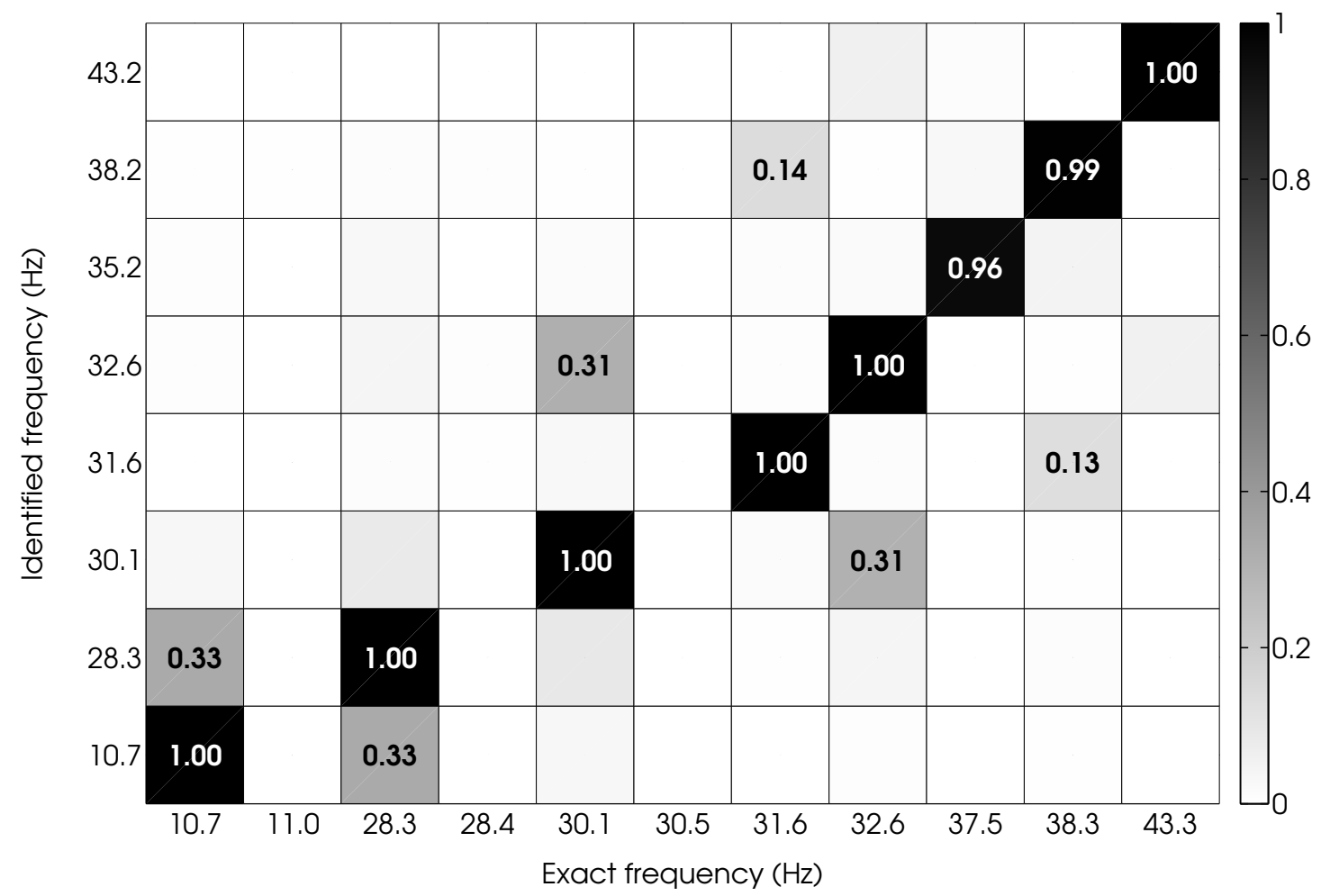

Figure 6: MACX matrix computed at order 18 using all measured channels. Modes 2, 4 and 6 at 11.0, 28.4 and $30.5 \mathrm{~Hz}$, respectively, are not identifiable from the measured data as they were excited perpendicularly to their deformed shapes. Moreover, non-negligible off-diagonal terms can be attributed to spatial aliasing in the reduced-order model of the SmallSat.

using the exact value of the associated clearance which is obviously questionable. The nonparametric approach to nonlinearity characterisation proposed in Section IV.B will show how this need for prior knowledge can be avoided. The real part of the coefficient exhibits almost no visible dependence upon frequency and its imaginary part remains more than 2 orders of magnitude smaller. These two observations are signs of an accurate estimation. The first line of Table 5 gives the averaged value of the nonlinear coefficient over $5-50$ $\mathrm{Hz}$, the relative error and the ratio between real and imaginary parts in logarithmic scaling. One points out the relatively low computational demand of the FNSI approach resulting from the possible discrimination of frequency samples down to the input band. 

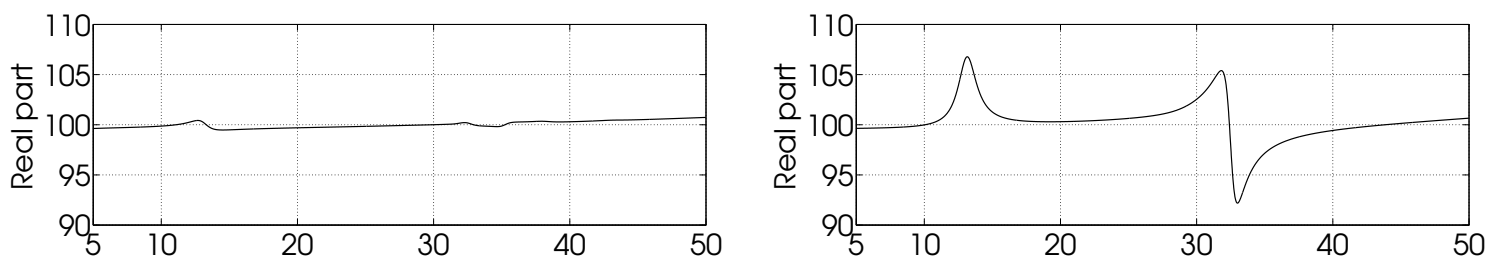

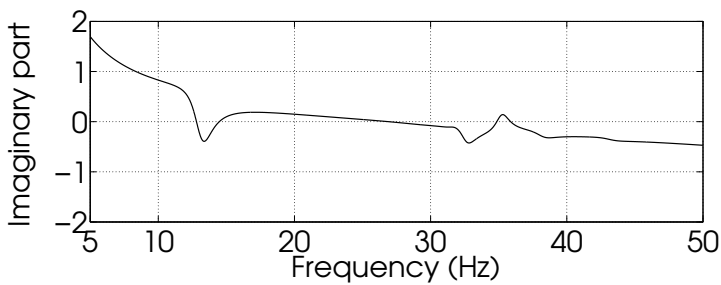

(a)

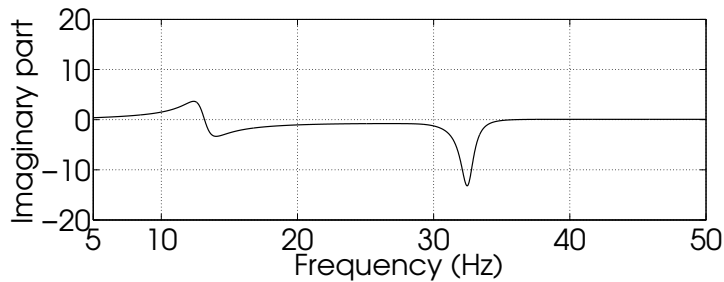

(b)

Figure 7: Complex-valued estimates of the $\mathrm{NC} 2-\mathrm{Z}$ nonlinear coefficient computed using (a) all measured channels and (b) three channels and represented over $5-50 \mathrm{~Hz}$.

\begin{tabular}{cccc}
\hline Real part & Error $(\%)$ & $\log _{10}$ (Real/Imag.) & Time $(s)$ \\
100.03 & 0.03 & 3.21 & 119 \\
100.29 & 0.29 & 2.19 & 1.8 \\
\hline
\end{tabular}

Table 5: Estimates of the NC $2-\mathrm{Z}$ nonlinear coefficient computed using all measured channels (first line) and three channels (second line). Real part averaged over $5-50 \mathrm{~Hz}$, relative error (in \%), ratio between the real and imaginary parts (in logarithmic scaling) and computational time given an AMD Phenom triple-core $2.10 \mathrm{GHz}$ processor unit with 4 Go RAM.

A second analysis of the single nonlinearity case can now be carried out considering only three output sensors, namely the axial DOFs of both nodes of the NC 2 and of the inertia wheel node. This corresponds to the more practical situation where the number of available sensors is limited and where system responses are only recorded close to the nonlinearity. The index $i$ is herein chosen equal to 100 to compensate for the reduction of processed channels. The resulting stabilisation diagram constructed by FNSI is depicted in Figure 8 and reveals fewer modes than in Figure 5. Specifically, only modes 1, 3, 5 and 8 are now observable as they involve an axial motion of the WEMS device. Full stabilisation is achieved at order 8 and the relative errors on the estimated natural frequencies and damping ratios (in \%) together with the diagonal terms of the corresponding MACX matrix are given in Table 6 . The quality of the results remains excellent except for the damping estimation of mode 8 , which nevertheless consists predominantly in a rotation of the instrument panel.

\begin{tabular}{cccc}
\hline Mode & Error on $\omega(\%)$ & Error on $\epsilon(\%)$ & MACX \\
1 & 0.17 & -0.04 & 1.00 \\
3 & 0.02 & -0.35 & 1.00 \\
5 & 0.06 & -3.10 & 1.00 \\
8 & 0.02 & -7.34 & 1.00 \\
\hline
\end{tabular}

Table 6: Relative errors on the estimated linear natural frequencies and damping ratios (in \%) and diagonal MACX values computed at order 8 using three measured channels.

The updated estimation of the nonlinear coefficient is shown in Figure 7 (b) and numerical values are reported in the second line of Table 5. The somewhat limited number of analysed sensors is found to yield a significant decrease of the computational burden but deteriorates the accuracy of the identification through 


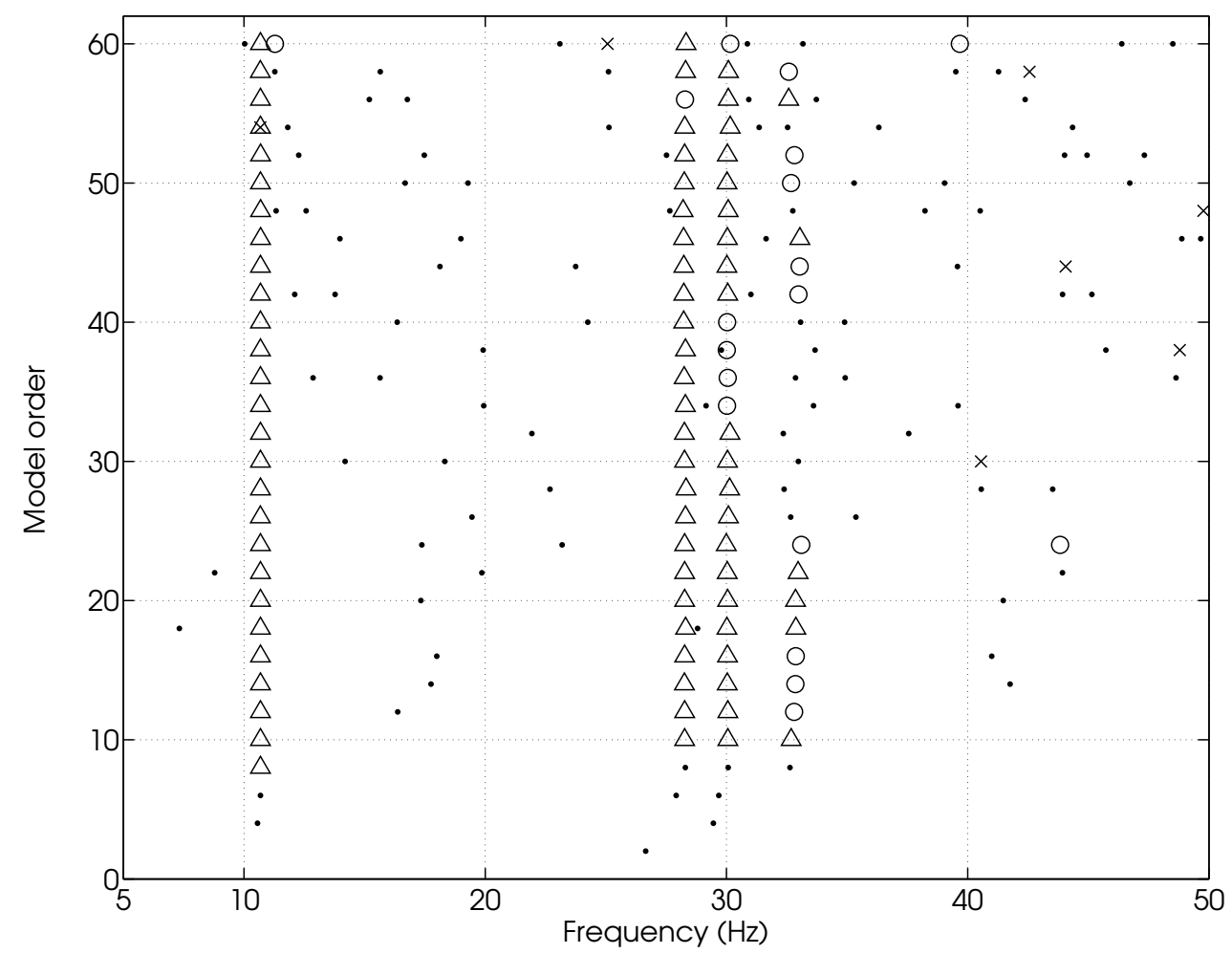

Figure 8: Stabilisation diagram computed by FNSI using three measured channels.

the appearance of peaks in the frequency-dependent nonlinear coefficient. These peaks coincide with antiresonances of the structure where the signal-to-noise ratio is low, hence increasing the sensitivity of the parameter estimation to modelling errors.

\section{IV.B. Nonparametric modelling of nonlinearities using cubic splines}

One of the major assets of the FNSI method is its ability to extract the underlying linear modal parameters of the system by means of a geometrical projection and without restriction as to the importance of nonlinearity in the system dynamics. However, this extraction assumes an adequate selection of the functional forms $g_{j}(q(t), \dot{q}(t))$ introduced in Equation (2). This task is challenging because nonlinearity may be caused by many different mechanisms and may result in plethora of dynamic phenomena. In the technical literature, the most standard practice is to resort to high-order global polynomial expansion, as in the work by Masri and Caughey ${ }^{14}$ where Chebyshev polynomials were exploited. The principle of polynomial expansion is to approximate the restoring force by a unique polynomial of some degree in displacement and velocity. However, in many circumstances, e.g. non-smooth or non-integer exponent-type nonlinearities, this approach may not be effective.

In this context, this section introduces an approximation strategy based on piecewise polynomials, or splines, which limits the need for having a priori information about the involved physical mechanisms. For the sake of simplicity, we restrict our scope to elastic nonlinearities $g_{j}(q(t))$. Let $q$ be divided into $L$ segments of arbitrary length and defined by their abscissas, denoted by $q_{k}$ for $k=1, \ldots, L+1$. Each abscissa is associated with an ordinate $g_{j, k}$, together defining a knot $\left(q_{k}, g_{j, k}\right)$ of the spline. Thus, if $q$ is a displacement value in between knots $k$ and $k+1$, the corresponding point of a third-order-spline-based approximation of $g_{j}(q(t))$ is given by

$$
g_{j}(q(t))=\left(2 t^{3}-3 t^{2}+1\right) g_{j, k}+\left(-2 t^{3}+3 t^{2}\right) g_{j, k+1}+\left(t^{3}-2 t^{2}+t\right)\left(q_{k+1}-q_{k}\right) g_{j, k}^{\prime}+\left(t^{3}-t^{2}\right)\left(q_{k+1}-q_{k}\right) g_{j, k+1}^{\prime}
$$

where $t$ is the normalised displacement $\frac{q-q_{k}}{q_{k+1}-q_{k}}$. The computation of the first derivatives $g_{j, k}^{\prime}=\partial g_{j, k} / \partial q_{k}$ 
can be achieved by forcing the cubic spline and its first two derivatives to be continuous across each of the interior knots. This results in $L-1$ linear constraint equations

$$
\frac{g_{j, k-1}^{\prime}}{q_{k}-q_{k-1}}+2\left(\frac{1}{q_{k}-q_{k-1}}+\frac{1}{q_{k+1}-q_{k}}\right) g_{j, k}^{\prime}+\frac{g_{j, k+1}^{\prime}}{q_{k+1}-q_{k}}=3\left(\frac{g_{j, k}-g_{j, k-1}}{\left(q_{k}-q_{k-1}\right)^{2}}+\frac{g_{j, k+1}-g_{j, k}}{\left(q_{k+1}-q_{k}\right)^{2}}\right) .
$$

As it is known that a nonlinear restoring force computed through the FNSI method should be zero and have zero slope at equilibrium, one should also enforce that, in the segment within which the abscissa of the equilibrium point lies,

$$
\left(t_{0}^{3}-2 t_{0}^{2}+t_{0}\right)\left(q_{k+1}-q_{k}\right) g_{j, k}^{\prime}+\left(t_{0}^{3}-t_{0}^{2}\right)\left(q_{k+1}-q_{k}\right) g_{j, k+1}^{\prime}=-\left(2 t_{0}^{3}-3 t_{0}^{2}+1\right) g_{j, k}-\left(-2 t_{0}^{3}+3 t_{0}^{2}\right) g_{j, k+1}
$$

and

$$
\left(3 t_{0}^{2}-4 t_{0}+1\right)\left(q_{k+1}-q_{k}\right) g_{j, k}^{\prime}+\left(3 t_{0}^{2}-2 t_{0}\right)\left(q_{k+1}-q_{k}\right) g_{j, k+1}^{\prime}=6\left(t_{0}-t_{0}^{2}\right)\left(g_{j, k}-g_{j, k+1}\right)
$$

where $t_{0}=\frac{-q_{k}}{q_{k+1}-q_{k}}$. Equations $(20),(21)$ and $(22)$ constitute $L+1$ equations that uniquely define the $L+1$ parameters $g_{j, 1}^{\prime}, \ldots, g_{j, L+1}^{\prime}$ as functions of the ordinates of the knots $g_{j, 1}, \ldots, g_{j, L+1}$. The first derivatives can then be substituted in Equation (19) to compute the basis functions associated with the ordinates that are eventually considered as input terms of the FNSI algorithm, as described in Section II.

This nonparametric modelling methodology can be applied to the SmallSat identification, using as previously only three channels in output. The NC $2-\mathrm{Z}$ relative displacement range is divided into 13 nonequally-width intervals, among which 10 intervals lie in the 10-\% neighbourhood of the clearances to improve the fitting of the discontinuity. This uneven discretisation assumes some prior knowledge about the exact location of the clearances that can be gained via different techniques, e.g. the restoring force surface method. ${ }^{14}$

From Figure 9, one selects the order 8 despite the absence of stabilisation in damping ratio of the fourth mode around $32 \mathrm{~Hz}$. This however trades off with a low variability of the identified model and subsequently with an improved stability of the nonliear coefficients versus frequency. The estimations of the modal properties at order 8 are listed in Table 7 . The reconstruction of the NC $2-\mathrm{Z}$ nonlinear stiffness curve is finally compared with the exact curve in Figures $10(\mathrm{a}-\mathrm{b})$ and proves that cubic splines, unlike polynomials, are reasonably well suited for representing non-smooth behaviour. One notes that cubic splines are also highly versatile as they can be utilised to model smooth restoring forces in contrast with first-order piecewise approximations.

\begin{tabular}{cccc}
\hline Mode & Error on $\omega(\%)$ & Error on $\epsilon(\%)$ & MACX \\
1 & 0.02 & -5.45 & 1.00 \\
3 & -0.15 & -5.62 & 1.00 \\
5 & 0.44 & 3.00 & 1.00 \\
8 & -3.70 & -5.79 & 1.00 \\
\hline
\end{tabular}

Table 7: Relative errors on the estimated linear natural frequencies and damping ratios (in \%) and diagonal MACX values computed at order 8 using cubic splines to model the NC $2-\mathrm{Z}$ nonlinearity and three measured channels. 


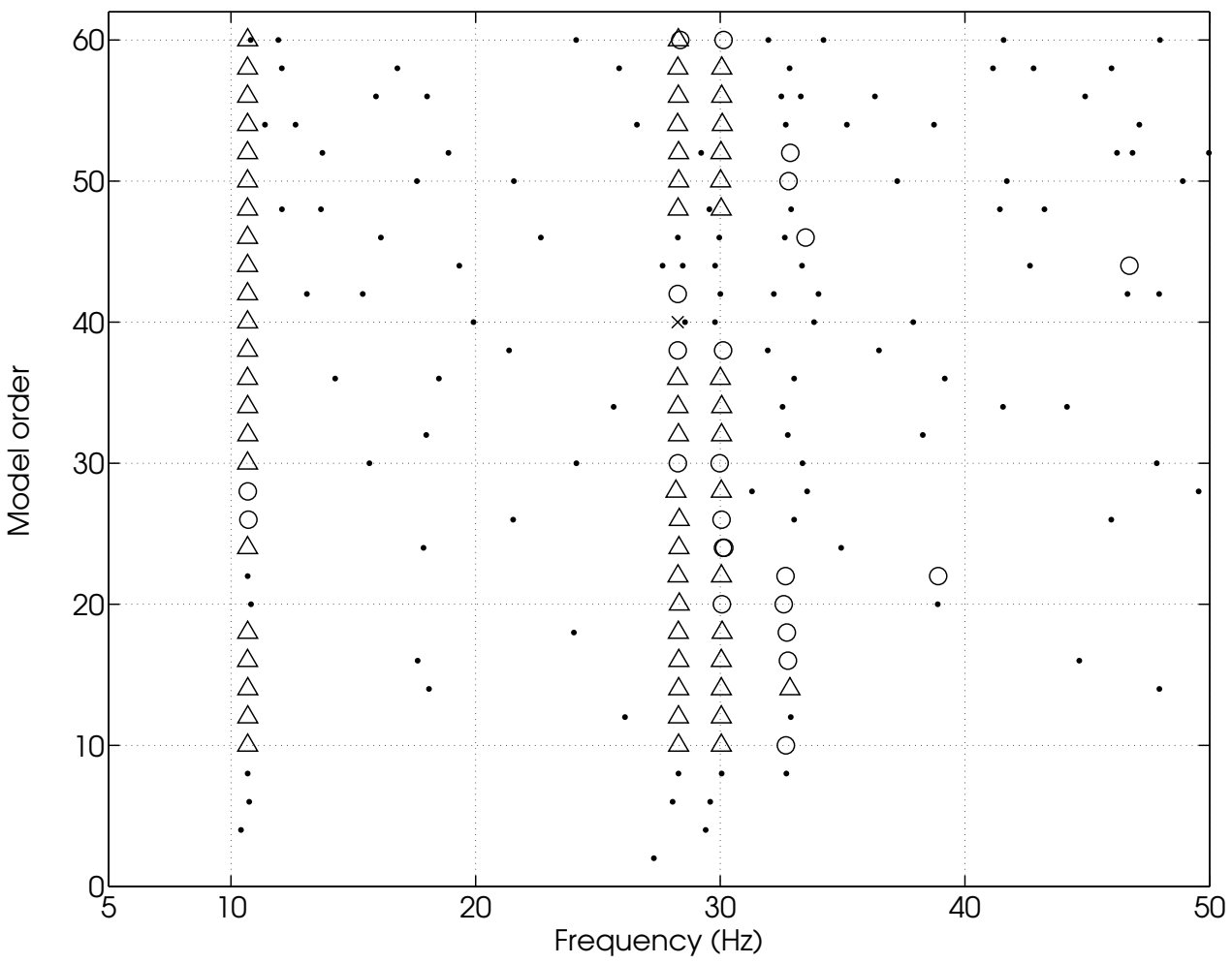

Figure 9: Stabilisation diagram computed using cubic splines to model the NC 2 - Z nonlinearity and three measured channels.

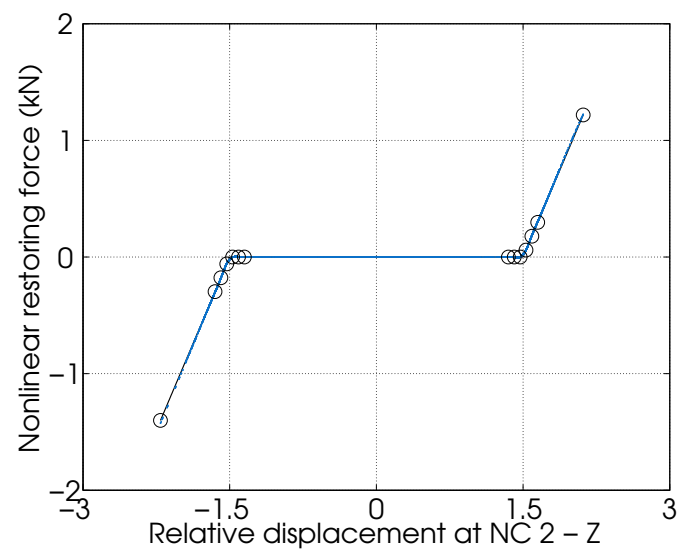

(a)

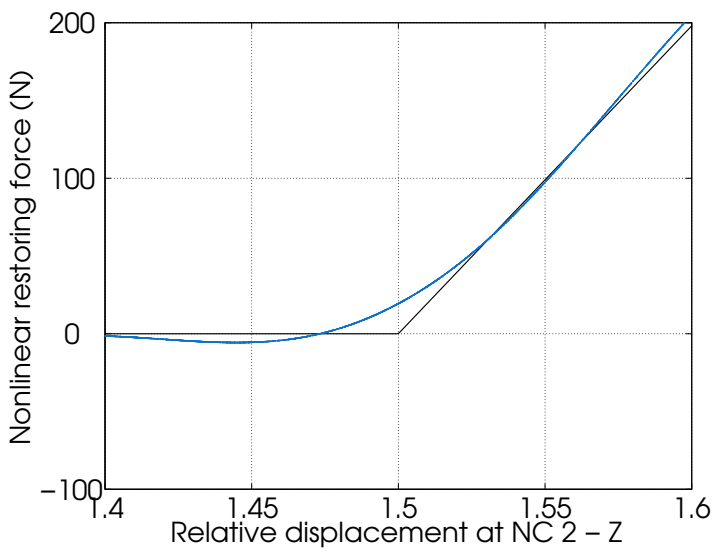

(b)

Figure 10: Exact nonlinear stiffness curve at the NC $2-\mathrm{Z}$ (in black) and FNSI reconstruction using cubic splines (in blue). (a) Entire stiffness curve where circles localise the chosen knots; (b) close-up of the discontinuity in positive displacement. 


\section{IV.C. Multiple nonlinearity case}

A periodic noise forcing with a RMS value of $200 N$ was applied to the inertia wheel node in the symmetric X - Y direction and caused the strong and comparable activation of all WEMS nonlinearities, as detailed in Table 8. As in the previous numerical experiment, the excitation was generated as the repetition of a Gaussian white-noise signal of 30,000 points and band-limited in $5-50 \mathrm{~Hz}$ to yield a total number of 240,000 points. Analysed samples were chosen similarly to the single nonlinearity case, namely 3,240 leakage-free frequency samples, and the index $i$ was set to 50 in the construction of the data matrices. One eventually opted for 18 channels in output, namely both sides of each NC (16) and the displacement of the inertia wheel node in the excitation and axial directions (2). The eight nonlinearities are represented using cubic splines, considering 9 intervals per nonlinear force, 6 of which being located in the 10-\% neighbourhood of the discontinuities.

\begin{tabular}{lcccc}
\hline & NC 1 & NC 2 & NC 3 & NC 4 \\
$\mathrm{X}$ & 13,453 & 13,001 & - & - \\
$\mathrm{Y}$ & - & - & 13,600 & 14,803 \\
$\mathrm{Z}$ & 28,043 & 21,218 & 25,854 & 31,661 \\
\hline
\end{tabular}

Table 8: Number of clearance exceedances. No entry means the absence of mechanical stop.

The inspection of the stabilisation diagram in Figure 11 indicates that the order 10 is adequate to capture the 5 first linear modes of the structure and stabilise the associated frequencies, damping ratios and modal shapes. The parameter estimation results given in Table 9 show that FNSI almost perfectly recovers the linear natural frequencies of the spacecraft from strongly nonlinear measurements, while the estimation of the damping ratios is also satisfactory. Figures $12(\mathrm{a}-\mathrm{f})$ finally depict the FNSI reconstruction of the nonlinear stiffness curves together with the corresponding exact curves. Lateral nonlinearities in the $\mathrm{X}$ and $\mathrm{Y}$ directions are superimposed in Figures $12(\mathrm{a}-\mathrm{b})$, respectively, as they cannot be distinguished owing to the symmetry of the WEMS device and of the external forcing. All this reasserts the reliable modelling capabilities of splines in the presence of non-smooth nonlinearities. One could also argue that most of the existing identification methods in the technical literature would be bound to failure in finding an accurate solution of such a high-dimensional inverse problem, namely encompassing 1 external force, 80 nonlinear basis functions and 18 outputs.

\begin{tabular}{cccc}
\hline Mode & Error on $\omega(\%)$ & Error on $\epsilon(\%)$ & MACX \\
1 & 0.39 & -1.35 & 1.00 \\
2 & 0.59 & 0.01 & 1.00 \\
3 & 0.06 & -2.73 & 1.00 \\
4 & 0.38 & -0.87 & 1.00 \\
5 & 0.79 & 4.43 & 0.99 \\
\hline
\end{tabular}

Table 9: Relative errors on the estimated linear natural frequencies and damping ratios (in \%) and diagonal MACX values computed at order 10 using cubic splines to model the eight nonlinearities. 


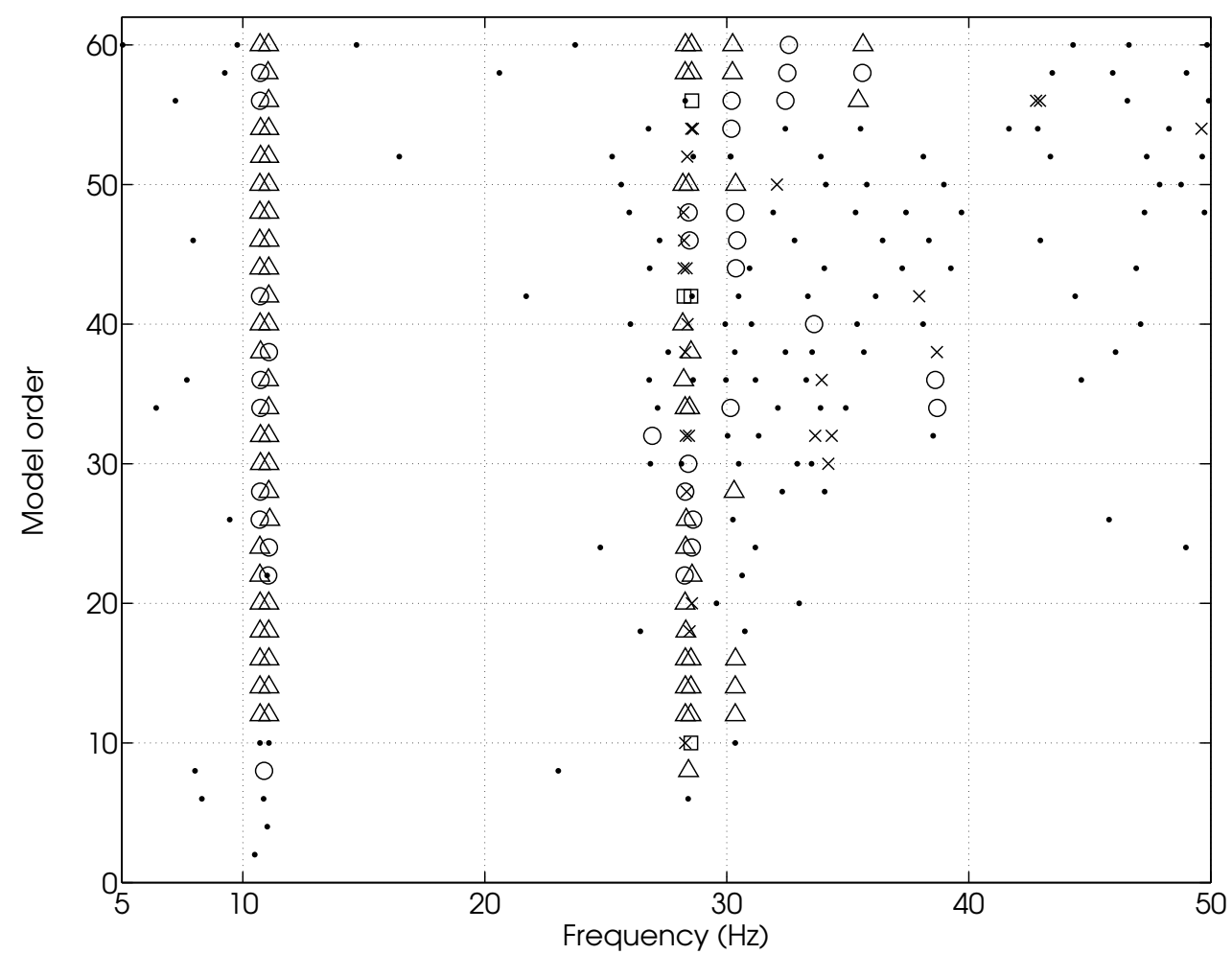

Figure 11: Stabilisation diagram computed using cubic splines to model the eight nonlinearities.

\section{Conclusions}

This paper aimed at exploiting a nonlinear generalisation of subspace methods formulated in the frequency domain, referred to as the FNSI method, to address the identification of the strongly nonlinear SmallSat structure. The coefficients of the nonlinear internal forces and the parameters of the underlying linear structure were shown to be accurately and rapidly estimated by this approach. Moreover, since the characterisation of nonlinearities is known to be of utmost importance in view of system identification while a priori knowledge is often limited, the paper also introduced a nonparametric modelling approach based on cubic splines which yielded promising results. This study is arguably one the first successful identifications of such a complex real-life nonlinear structure assuming little prior knowledge about the involved nonlinear mechanisms.

Additional investigations are needed to further assess the capabilities of the FNSI method. In particular, the variability of the identification over repeated experiments due to the random nature of the excitation and the noise should be carefully analysed. A specific attention should also be devoted to the asymptotic behaviour of the proposed subspace algorithm for an increasing number of measured samples and block rows. Finally, the robustness of the cubic-spline-based representation of nonlinearities would also be worth assessing. An automatic selection of the number of sub-intervals or iterative approximations considering an adaptive refinement strategy would be other important advances.

\section{Acknowledgements}

The author J.P. Noël would like to acknowledge the Belgian National Fund for Scientific Research (FRIA fellowship) for its financial support. The authors also thank Astrium SAS for sharing information about the SmallSat spacecraft. 


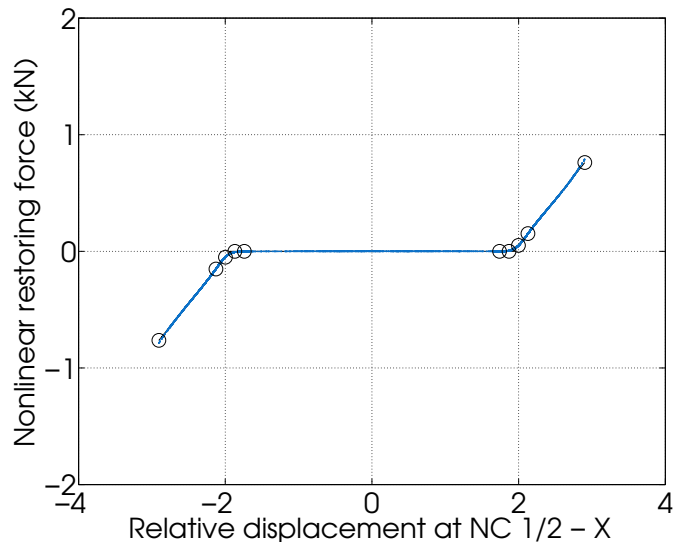

(a)

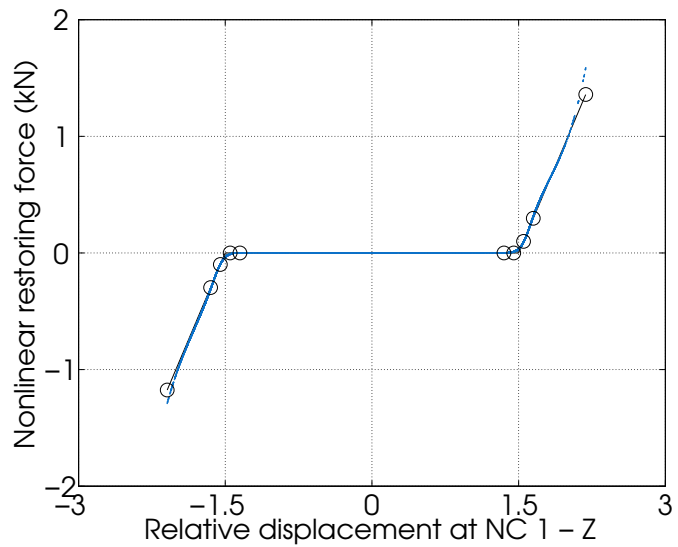

(c)

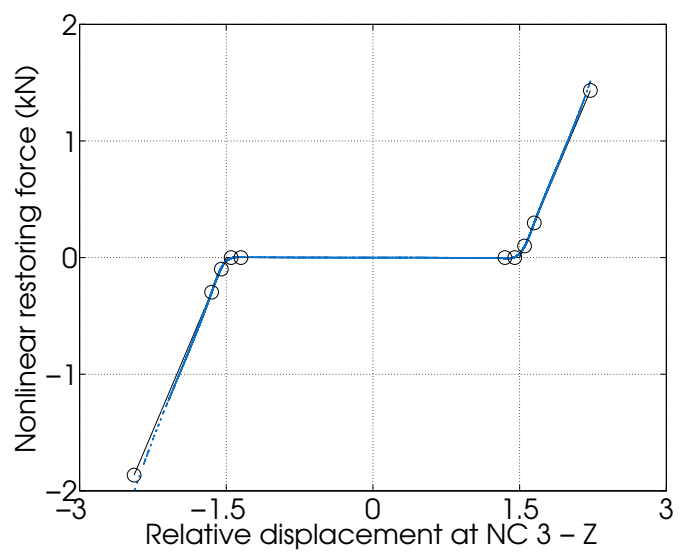

(e)

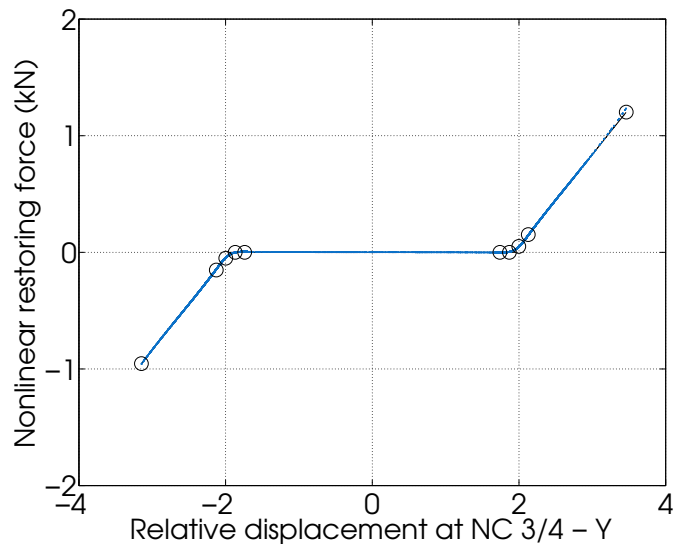

(b)

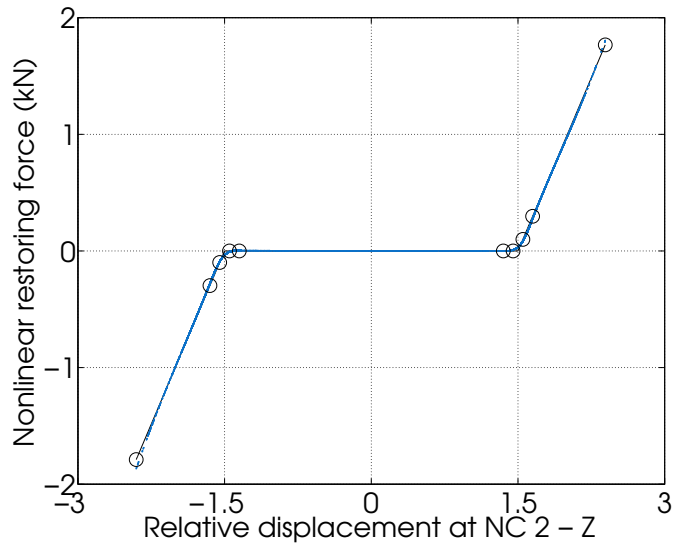

(d)

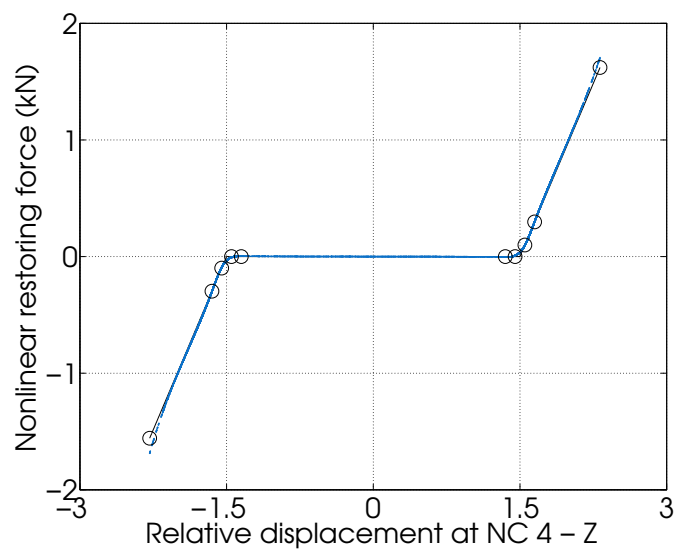

(f)

Figure 12: Exact nonlinear stiffness curves (in black) and FNSI reconstruction using cubic splines (in blue). (a) NC $1-\mathrm{X}$ and NC $2-\mathrm{X}$; (b) NC $3-\mathrm{Y}$ and NC $4-\mathrm{Y}$; (c) NC $1-\mathrm{Z}$; (d) NC 2 - Z; (e) NC 3 - Z; (d) $\mathrm{NC} 4-\mathrm{Z}$. 


\section{References}

${ }^{1}$ Van Overschee, P., De Moor, B., Subspace Identification for Linear Systems, Kluwer Academic Publishers, Boston/London/Dordrecht, 1996.

${ }^{2}$ Reynders, E., De Roeck, G., "Reference-based combined deterministic-stochastic subspace identification for experimental and operational modal analysis", Mechanical Systems and Signal Processing, Vol. 22, 2008, pp. 617-637.

${ }^{3}$ Mevel, L., Hermans, L., Van Der Auweraer, H., "Application of a subspace-based fault detection method to industrial structures", Mechanical Systems and Signal Processing, Vol. 13, 1999, pp. 823-838.

${ }^{4}$ Lacy, S. L., Bernstein, D. S., "Subspace identification for non-linear systems with measured-input non-linearities", International Journal of Control, Vol. 78, 2005, pp. 906-926.

${ }^{5}$ Marchesiello, S. , Garibaldi, L., "A time domain approach for identifying nonlinear vibrating structures by subspace methods", Mechanical Systems and Signal Processing, Vol. 22, 2008, pp. 81-101.

${ }^{6}$ Noël, J.P., Kerschen, G. "A new subspace-based approach to identify nonlinear mechanical structures in the frequency domain", Proceedings of the 16th IFAC Symposium on System Identification, The International Federation of Automatic Control, Brussels, Belgium, 2012.

${ }^{7}$ Kerschen, G., Worden, K., Vakakis, A. F., Golinval, J.C., "Past, present and future of nonlinear system identification in structural dynamics", Mechanical Systems and Signal Processing, Vol. 20, 2006, pp. 505-592.

${ }^{8}$ Adams, D. E., Allemang, R. J., "A frequency domain method for estimating the parameters of a non-linear structural dynamic model through feedback", Mechanical Systems and Signal Processing, Vol. 14, No. 4, 2000, pp. 637-656.

${ }^{9}$ Pintelon, R., Schoukens, J., Subspace Identification for Linear Systems, IEEE Press, Piscataway, 2001.

${ }^{10}$ Van Overschee, P., De Moor, B., "Continuous-time frequency domain subspace system identification", Signal Processing, Vol. 52, 1996, pp. 174-194.

${ }^{11}$ Russell, A. G., "Thick skin, faceted, CFRP, monocoque tube structure for smallsats", Proceedings of the European Conference on Spacecraft Structures, Materials and Mechanical Testing, European Space Agency, Noordwijk, The Netherlands, 2000.

${ }^{12}$ Peeters, B., Climent, H., de Diego, R., de Alba, J., Ahlquist, J. R., Carreo, J. M., Hendricx, W., Rega, A., Garcia, G., Deweer, J., Debille, J., "Modern solutions for Ground Vibration Testing of large aircraft", Proceedings of the International Modal Analysis Conference XXVI, Society for Experimental Mechanics, Orlando, FL, 2008.

${ }^{13}$ Vacher, P., Jacquier, B., Bucharles, A., "Extensions of the MAC criterion to complex modes", Proceedings of the ISMA 2010 International Conference on Noise and Vibration Engineering, Leuven, Belgium, 2010.

${ }^{14}$ Masri, S. F., Caughey, T. K., "A nonparametric identification technique for nonlinear dynamic problems", Journal of Applied Mechanics, Vol. 46, 1979, pp. 433-447. 